\title{
Lineage of origin in rhabdomyosarcoma informs pharmacological response
}

\author{
Jinu Abraham, ${ }^{1}$ Yaiza Nuñez-Álvarez, ${ }^{2}$ Simone Hettmer, ${ }^{3,4}$ Elvira Carrió, ${ }^{2}$ Hung-I Harry Chen, ${ }^{5}$ \\ Koichi Nishijo, ${ }^{5}$ Elaine T. Huang, ${ }^{1}$ Suresh I. Prajapati, ${ }^{5}$ Robert L. Walker, ${ }^{6}$ Sean Davis, ${ }^{6}$ \\ Jennifer Rebeles, ${ }^{5}$ Hunter Wiebush, ${ }^{5}$ Amanda T. McCleish, ${ }^{5}$ Sheila T. Hampton, ${ }^{5}$ \\ Christopher R.R. Bjornson, ${ }^{7}$ Andrew S. Brack, ${ }^{7}$ Amy J. Wagers, ${ }^{3}$ Thomas A. Rando, ${ }^{7}$
} Mario R. Capecchi, ${ }^{8}$ Frank C. Marini, ${ }^{9}$ Benjamin R. Ehler, ${ }^{10}$ Lee Ann Zarzabal, ${ }^{10}$ Martin W. Goros, ${ }^{10}$ Joel E. Michalek, ${ }^{10}$ Paul S. Meltzer, ${ }^{6}$ David M. Langenau, ${ }^{11}$ Robin D. LeGallo, ${ }^{12}$ Atiya Mansoor, ${ }^{13}{ }^{10}$ Yidong Chen, ${ }^{5,10}$ Mònica Suelves, ${ }^{2}$ Brian P. Rubin, ${ }^{14}$ and Charles Keller ${ }^{1,15}$

${ }^{1}$ Pediatric Cancer Biology Program, Papé Family Pediatric Research Institute, Department of Pediatrics, Oregon Health and Science University, Portland, Oregon 97239, USA; ${ }^{2}$ Institute of Predictive and Personalized Medicine of Cancer (IMPPC), Germans Trias i Pujol Health Sciences Research Institute (IGTP), 08916 Badalona, Barcelona, Spain; ${ }^{3}$ Howard Hughes Medical Institute, Department of Stem Cell and Regenerative Biology, Harvard University, Harvard Stem Cell Institute, Joslin Diabetes Center, Cambridge, Massachusetts 02138, USA; ${ }^{4}$ Department of Pediatric Oncology, Dana Farber Cancer Institute, Division of Pediatric Hematology/Oncology, Children's Hospital, Boston, Massachusetts 02115, USA; ${ }^{5}$ Greehey Children's Cancer Research Institute, University of Texas Health Science Center, San Antonio, Texas 78229, USA; ${ }^{6}$ Oncogenomics Section, Pediatric Oncology Branch, Advanced Technology Center, National Cancer Institute, Gaithersburg, Maryland 20877, USA; ${ }^{7}$ Department of Neurology, Department of Neurological Sciences, Glenn Laboratories for the Biology of Aging, Stanford University, Palo Alto, California 94304, USA; ${ }^{8}$ Department of Human Genetics, University of Utah, Salt Lake City, Utah 84112, USA; ${ }^{9}$ Institute for Regenerative Medicine, Wake Forest School of Medicine, Winston-Salem, North Carolina 27157, USA; ${ }^{10}$ Department of Epidemiology and Biostatistics, University of Texas Health Science Center, San Antonio, Texas 78229, USA; ${ }^{11}$ Department of Pathology, Center for Cancer Research, Massachusetts General Hospital, Charlestown, Massachusetts 02129, USA; ${ }^{12}$ Department of Pathology, University of Virginia, Charlottesville, Virginia 22903, USA; ${ }^{13}$ Department of Pathology, Oregon Health and Science University, Portland, Oregon 97239, USA; ${ }^{14}$ Department of Anatomic Pathology, Department of Molecular Genetics, Taussig Cancer Center, Lerner Research Institute, Cleveland Clinic Foundation, Cleveland, Ohio 44195, USA

Lineage or cell of origin of cancers is often unknown and thus is not a consideration in therapeutic approaches. Alveolar rhabdomyosarcoma (aRMS) is an aggressive childhood cancer for which the cell of origin remains debated. We used conditional genetic mouse models of aRMS to activate the pathognomonic Pax3:Foxo1 fusion oncogene and inactivate $p 53$ in several stages of prenatal and postnatal muscle development. We reveal that lineage of origin significantly influences tumor histomorphology and sensitivity to targeted therapeutics. Furthermore, we uncovered differential transcriptional regulation of the Pax3:Foxo1 locus by tumor lineage of origin, which led us to identify the histone deacetylase inhibitor entinostat as a pharmacological agent for the potential conversion of Pax3:Foxo1-positive aRMS to a state akin to fusion-negative RMS through direct transcriptional suppression of Pax3:Foxo1.

[Keywords: alveolar rhabdomyosarcoma; Pax3:Foxo1; sarcoma; satellite cell; myoblast; histone]

Supplemental material is available for this article.

Received January 22, 2014; revised version accepted June 3, 2014.

Rhabdomyosarcoma is an aggressive solid tumor for which the cell lineage of origin of alveolar rhabdomyosarcoma (aRMS) remains debated (Tiffin et al. 2003; Charytonowicz et al. 2009; Hettmer and Wagers 2010; Hettmer et al. 2011). Whereas the adult pleomorphic subtype is felt to originate

\footnotetext{
${ }^{15}$ Corresponding author: E-mail keller@ohsu.edu

Article is online at http://www.genesdev.org/cgi/doi/10.1101/gad.238733.114 Freely available online through the Genes \& Development Open Access option.
}

from muscle stem cells (satellite cells) (Hettmer et al. 2011; Blum et al. 2013), the embryonal subtype has been attributed to arise more often from myoblasts than upstream myogenic progenitors (i.e., satellite cells), the latter of which are more prone to transform into undifferentiated pleomorphic sarcomas (UPSs) having relatively little myodifferentiation potential (Rubin et al. 2011). Even more

(c) 2014 Abraham et al. This article, published in Genes \& Development, is available under a Creative Commons License (Attribution $4.0 \mathrm{In}$ ternational), as described at http://creativecommons.org/licenses/by/4.0. 
intriguing are possible nonmyogenic cells of origin of embryonal rhabdomyosarcoma (eRMS) from the adipogenic lineage (Hatley et al. 2012; Kikuchi and Keller 2012). For the alveolar subtype that is often incurable when metastatic (Malempati and Hawkins 2012; Hawkins et al. 2013), we previously suggested Myf6-expressing (differentiating) cells of the myogenic lineage as the cell of origin (Keller et al. 2004a,b). These studies had used conditional mouse models triggering in the Myf6Cre lineage the pathognomonic Pax3:Foxo1 chimeric oncogene, which is commonly found in the human disease as a result of a t(2;13) translocation (Arndt and Crist 1999). Nevertheless, we and others have reconsidered whether mesenchymal stem cells or satellite cells could be an alternate cell of origin (Ren et al. 2008; Charytonowicz et al. 2009; Hettmer and Wagers 2010), and thus we performed the studies described here.

An interesting aspect of the search for cell of origin is that we uncovered a differential susceptibility of the Pax3:Foxo1 locus to be transcribed based on the cell lineage originally transformed. This finding may have translational significance in that the related Pax 7 locus in myogenic progenitors is a classic example of a bivalent epigenetic locus (Mozzetta et al. 2011), with different transcriptional activation states depending on degree of myodifferentiation, and thus is potentially amenable to pharmacological intervention. Transcription factors have not typically been considered approachable therapeutic targets, but if transcription of Pax3:Foxo1 itself could be inhibited, then the implicit conversion of fusionpositive aRMS to fusion-negative RMS would have great appeal, given the substantial difference in outcomes between these two clinical groups in retrospective studies (Sorensen et al. 2002; Missiaglia et al. 2012).

\section{Results}

\section{The p53 pathway is frequently aberrant in aRMS}

We and others have commonly used p53 inactivation in mouse models of aRMS (Keller et al. 2004a), yet the clinical relevance of $p 53$ deregulation at the genetic and/or functional pathway levels is debated (Takahashi et al. 2004; Ognjanovic et al. 2012). To address this issue, we used metagene analysis to test whether $p 53$ functional inactivation was a clinically relevant cooperative initiating mutation in aRMS (Supplemental Fig. S1A). Using our previously described metagene analysis and S-score method (Rubin et al. 2011), we analyzed a global gene expression data set (Supplemental Tables 1, 2) of 62 PAX3:FOXO1 ${ }^{+}$and $24 \mathrm{PAX} 7: \mathrm{FOXO1}^{+}$human aRMS tumors for aberrant signaling of the rhabdomyosarcoma-associated p53 pathway. We found that $85 \%$ of $\mathrm{PAX} 3: \mathrm{FOXO1}^{+}$tumors exhibited a gene expression signature consistent with the "p53 off" state. Similarly, 75\% of PAX7:FOXO1 tumors also had a p53 off state. Thus, the p53 off state was a common signature in human samples.

Pax3:Foxo1 leads to forms of sarcoma for satellite cells different from any other myogenic lineage

Having established a prominent role for p53 pathway inactivation and Pax3:Foxol activation as driver events in the genesis of aRMS, we generated mouse models (Keller et al. 2004a) to mimic these initiating driver events through targeting to specific muscle cell types in fetal and postnatal development (Fig. 1A). Tumors occurred in embryonic muscle lineages (MCre for hypaxial Pax3) and embryonic and fetal muscle lineages (Myf6Cre for Myf6) as well as the postnatal satellite cell lineage (Pax7CreER) (Fig. 1B,C). For the latter, tamoxifen was administered at $30 \mathrm{~d}$ of age (adolescence). The Myf5Cre lineage was embryonic-lethal (usually resulting in exencephaly) (data not shown) in all litters except for one animal (U24014) that later went on to develop a tumor of the lower extremity at $74 \mathrm{~d}$ of age. The most susceptible lineages were fetal myogenic progenitors and postnatal satellite cells but not Myf5- or Myf6-expressing postnatal committed myogenic progenitors: Of 18 Myf5CreER, Pax3:Foxo1,p53 mice given tamoxifen at $30 \mathrm{~d}$ of life (P30 [postnatal day 30]) and observed 43-510 d (median, 395 ), only one developed at age $401 \mathrm{~d}$ a tumor that was diagnosed as aRMS solid variant of the cranial muscle (Supplemental Fig. S1B-D). Similarly, from eight Myf6CreER, Pax3:Foxo1, p53 mice given tamoxifen at P30 and observed 232-505 d (median, 396), no mice developed tumors. Postnatal lineage tracing of the postnatal Myf6CreER lineage showed marking of myofibers as well as Pax $7^{+}$sublaminar satellite cells (Supplemental Fig. S2), a postnatal result complementing a report that embryonic Myf6Cre might prime Pax $7^{+}$satellite cells (Sambasivan et al. 2013). However, despite a recent report showing that Myf6Cre is active in early embryonic muscle progenitors that can become satellite cells (Sambasivan et al. 2013), other evidence for the Myf6Cre fetal myoblast population being a more common cell of origin for Pax3:Foxo $1^{+}$aRMS than a postnatal satellite cell includes (1) the absence of tumors from Myf6CreER, Pax3:Foxo1, p53 mice in which $\mathrm{Myf}^{+}$satellite cells can activate Pax3:Foxo1, (2) the absence of aRMS tumors from Pax7CreER, Pax3:Foxo1, p53 mice in which the $\operatorname{Pax} 7^{+}$satellite cell population at large can activate Pax3:Foxo1, and (3) the absence of aRMS tumors from Myf5CreER, Pax3:Foxo1, p53 mice whose nonquiescent satellite cells can activate Pax3: Foxo1 (Biressi et al. 2013).

To address the reported possibility that nonmyogenic, marrow-derived cells might give rise to aRMS-at least when Pax3:Foxo1 is driven by a nonnative promoter (Ren et al. 2008) - we first examined cells of the bone marrow compartment for the presence of cells derived from a Myf6Cre or Pax3CreKI (Pax3) (Engleka et al. 2005) ontogeny using eYFP (Keller et al. 2004a) and RFP (Vintersten et al. 2004) reporters, respectively. This lineage tracing was done without introducing Pax3:Foxo1 or p53 mutations. Neither the Pax3 nor the Myf6 lineage was represented in the bone marrow compartment in $>0.4 \%$ of mesenchymal or hematopoietic cells (Supplemental Fig. S1E,F), and thus this line of experimentation was discontinued.

At the histological level, the embryonic MCre (hypaxial Pax3Cre) and fetal Myf6Cre lineages led to aRMS histology (Fig. 1D). The single animal that survived past birth for the Myf5Cre lineage (U24014) also developed 
A

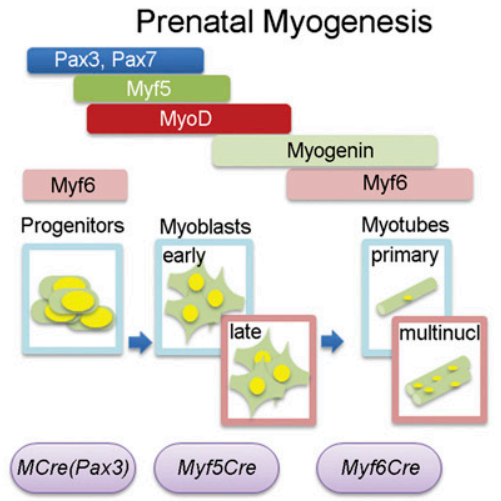

B

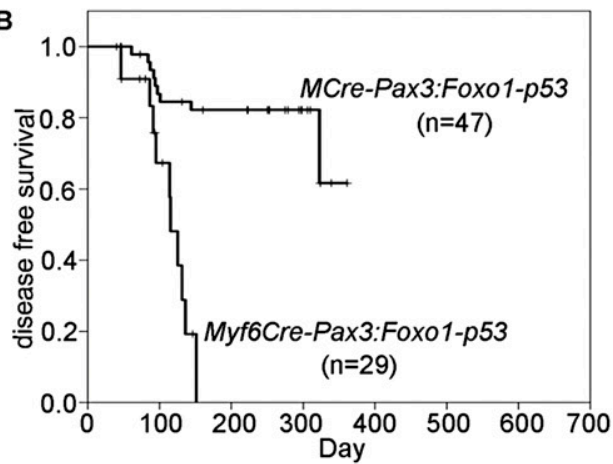

C

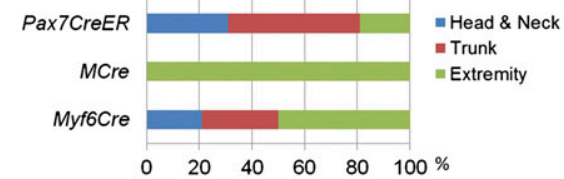

D
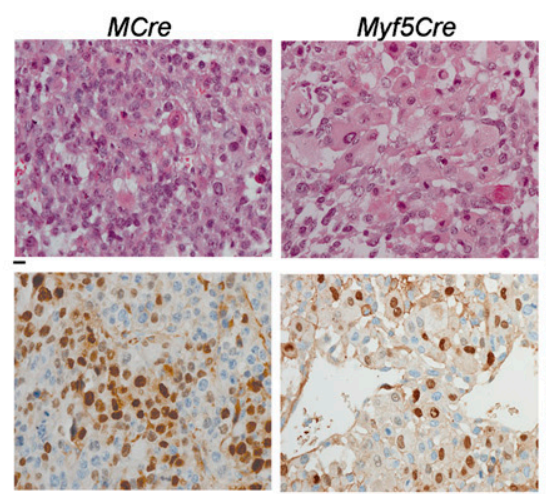

E
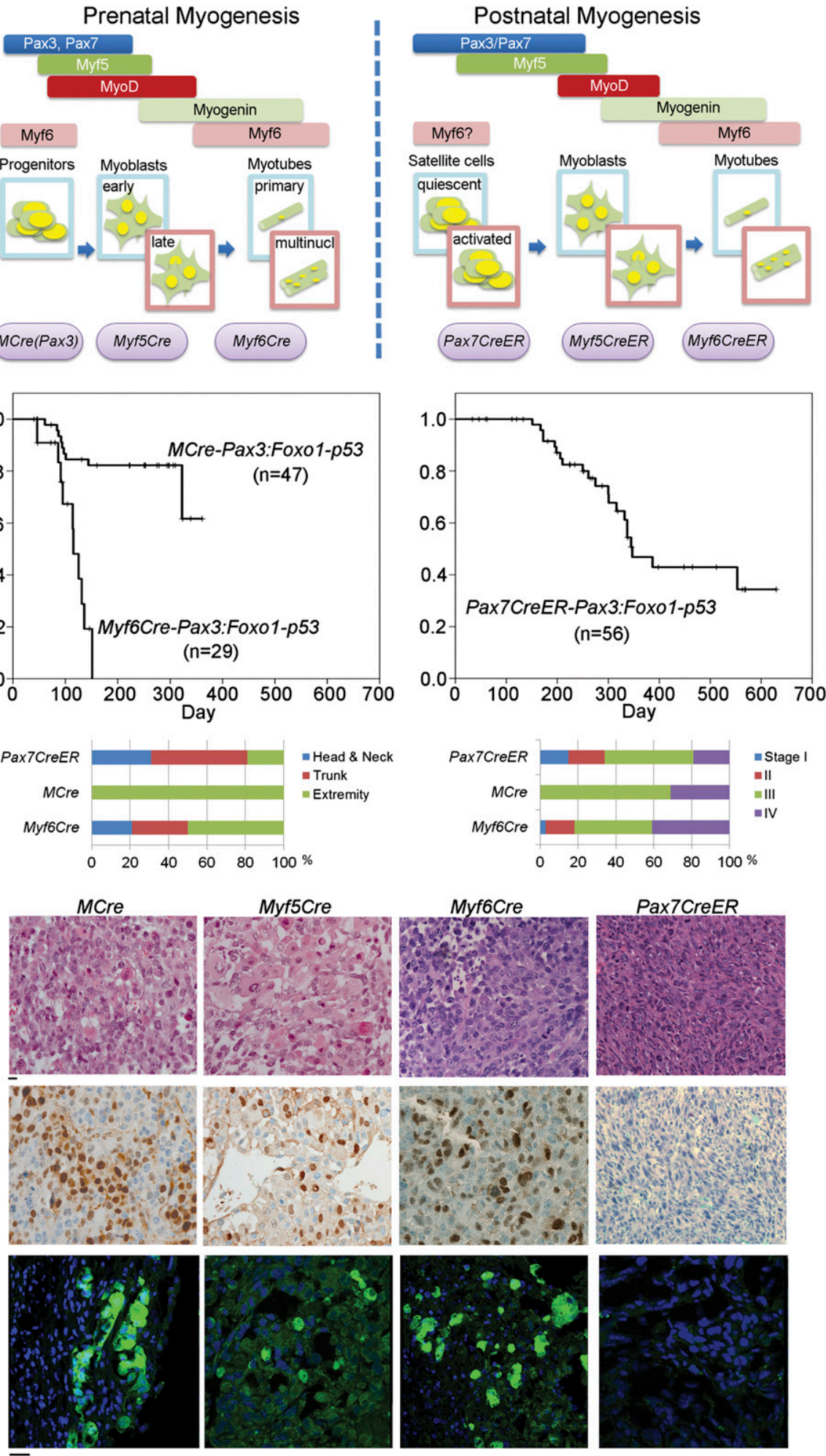

Figure 1. Characteristics of tumors from Pax7CreER, Myf5Cre, Myf6Cre, and MCre mice. (A) Representation of prenatal and postnatal myogenesis in the context of Cre drivers used to trigger tumors in this study. For Pax7CreER, Myf5CreER, and Myf6CreER mice, tamoxifen was administered at P30 (see the Materials and Methods). (multinucl.) Multinucleated. (B) Pax7CreER mice and MCre mice showed prolonged survival and lower tumor incidence compared with Myf6Cre mice (tumor incidences were $M C r e, 40 \%$; Pax7CreER, $38 \%$; and Myf6Cre, 100\% at $1 \mathrm{yr}$ after birth). Myf5Cre mice were embryonic-lethal (see the text). All mice also harbor homozygous $\mathrm{Pax} 3^{\mathrm{P} 3 \mathrm{Fm} / \mathrm{P} 3 \mathrm{Fm}}$ and $\mathrm{p} 53^{\mathrm{F} 2-10 / \mathrm{F} 2-10}$ alleles. $(C)$ Location and disease stages in each strain. Pax7CreER tumors were more frequently observed in head/neck and genital areas, whereas MCre mice developed tumors exclusively in the extremities. The highest frequency of stage IV metastasis (40\%) was seen for Myf6Cre mice. The IRSG staging system was scaled to mice such that a 4-mm-diameter mouse tumor was equated with a 5-cm-diameter human tumor (Nishijo et al. 2009a). ( $D$, top row) Histological analysis of tumors. Myf5Cre, Myf6Cre, and MCre tumors were small round cells (aRMS), whereas most Pax7CreER tumors showed spindle cell morphology (pleomorphic RMS/pleomorphic spindle cell sarcoma). (Bottom row) Immunohistochemistry for Myogenin. (E) eYFP protein expression representing the transcriptional activation status of Pax3:Foxo1 fusion gene was at the lower limit of detection in Pax7-CreER tumors. Bars, $10 \mu \mathrm{M}$. 
aRMS with numerous rhabdoid-type rhabdomyoblasts and diffuse anaplasia (Fig. 1D). In contrast, the Pax7CreER postnatal satellite cell lineage led to tumors diagnosed as pleomorphic RMS/pleomorphic spindle cell sarcoma by histological and immunohistochemical criteria (Fig. 1D). Surprisingly, the eYFP marker of Pax3:Foxo1 was expressed only weakly in Pax7CreER-derived tumors compared with the other tumors (Fig. 1E). Correspondingly, Pax3:Foxo1 expression by RT-PCR was variable and generally low in Pax7CreER tumors compared with mice bearing the Pax3:Foxo1 conditional alleles and other Cre drivers (Fig. 2A).

To begin exploring the potential causes of the low Pax3:Foxo1 expression from Pax7CreER,Pax3,p53 tumors, we evaluated the Cre/LoxP recombination efficiency at the Pax3 locus. By genotyping tumors, Pax3 locus recombination frequency was found to be lower in tumors of Pax7CreER-expressing satellite cell origin than Myf6Cre-expressing prenatal (presumably myoblast) origin. Generally Pax7CreER tumors had only one Pax3 conditional knock-in allele, $P 3 F m$, converted to the active Pax3:Foxo1 form, P3Fa (Fig. 2B; see the Discussion). In contrast, recombination for the $p 53$ locus by Pax7CreER in tumors was uniformly complete for both p53 alleles. Furthermore, the lower Pax3 recombination frequency alone would not account for the difference in histology because Pax3 $(\mathrm{P} 3 \mathrm{Fa} / W T)$ tumors from the Myf6Cre lineage presented with an aRMS histology, not a pleomorphic histology (Fig. 2C; Supplemental Table 3). By quantitative analysis, the mean florescence intensity of eYFP in primary tumor cell cultures derived from Pax7CreER,Pax3(P3Fm/P3Fa) tumors was rarely above the level of C2C12-negative control cells except in rare instances (Fig. 2D; see the Discussion). On the other hand, mean fluorescence intensity of eYFP in primary cell cultures derived from a Myf6Cre, $\mathrm{Pax} 3(\mathrm{P} 3 \mathrm{Fa} / \mathrm{P} 3 \mathrm{Fa}$ ) tumor was twofold to threefold higher than control C2C12 cells (Fig. 2D). RT-PCR studies also showed higher levels of Pax3:Foxo1 mRNA levels in murine primary tumor cell cultures derived from Myf6Cre,Pax3(P3Fa/P3Fa) tumors (Fig. 2E, green) compared with primary tumor cell cultures from Pax7CreER,Pax3(P3Fa/P3Fa) tumors (U31425 and U28285) (Fig. 2E). Altogether, these results suggest that inherently less transcription occurs from the Pax3 locus in Pax7CreER-derived tumors and decrease the probability that aRMS arises from $\operatorname{Pax} 7^{+}$postnatal satellite cells.

\section{Tumors arising from non-Pax7CreER cell of origin authentically recapitulate human aRMS}

To further evaluate the lineages that might give rise to aRMS, we compared expression of validated markers of Pax:Foxo1 ${ }^{+}$aRMS and eRMS (Wachtel et al. 2006). Myf6Cre myoblast-derived mouse tumors expressed significantly higher levels of the Pax3:Foxo1-specific aRMS marker Tcfap2b, whereas Pax7CreER satellite cell-derived tumors expressed reciprocally higher levels of the eRMS-specific marker Fbn2 (Fig. 3A). We then turned to principal component analysis (PCA) to compare Pax3:Foxo1-bearing tumors with those in the eRMS-UPS spectrum (Rubin et al. 2011). Results showed that Pax7CreER satellite cell-derived tumors were highly similar to eRMS/UPS tumor models that we previously reported (Fig. 3B; Rubin et al. 2011). In contrast, all Pax3:Foxo1-bearing tumors histologically consistent with aRMS from the MCre (Pax3), Myf5Cre, and Myf6Cre lineages formed an independent outgroup (Fig. 3B). Thus, the Pax7CreER postnatal satellite cell lineage did not appear to be poised for giving rise to aRMS when the Pax3:Foxo1 oncogene at the Pax3 locus is triggered; instead, cells of Pax7CreER satellite cell lineage had a tendency to give a spindle cell-like tumor phenotype. We next hypothesized that lineage-specific drug sensitivity could be best interrogated by an ontogeny series that would include Pax7CreER tumors for which Cre-mediated recombination or transformation occurred after the satellite cell state. From Figure 3B, we expected an intermediate state to be rare, but from the Pax3:Foxo1 data in Figure 2E (i.e., samples U28285, U31423, and U29416), we expected at least some instances to exist. To identify a potential ontogeny series, we first sought to find model primary cell cultures best representing Pax3:Foxo1-harboring satellite cell (Pax7CreER lineage)-derived tumors versus Pax3: Foxol-harboring myoblast (Myf6Cre lineage)-derived tumors. Comparison of gene expression for these two tumor types is given in Supplemental Table 4. The Pax7CreER lineage was associated with expression of Cav1, Dcn, and Cxcl12, whereas the Myf6Cre lineage was associated with expression of Sct, Stc1, and Ryr3 (Supplemental Table 4). We choose five primary cell cultures-i.e., U29415, U31425, U28285, U31423, and U29416-of Pax7CreER lineage and U20325, U23674, and U21459 of Myf6Cre lineage to test the expression of Cav1, Dcn, Cxcl12, Sct, Stc1, and Ryr3 by RT-PCR. These studies showed an interesting gradient pattern in which the expression of markers associated with Pax 7 CreER lineage was highest in U29415 cells followed by U31425 or U28285 and lower expression in $\mathrm{Myf} 6 \mathrm{Cre}$ lineage primary cell cultures U20325, U23674, and U21459. Conversely, the expression of markers associated with Myf6Cre lineage showed a reverse gradient pattern where the highest expression was observed in U21459/U23674, followed by U29416 or U20325, U31423, U28285, U31425, and U29415 cells (Supplemental Fig. S3A). These results suggested that U31425, U28285, U31423, and U29416 represent the "intermediate" myodifferentiation and transformation between U29415 (Pax7CreER) and U23674/U21459 (Myf6Cre) (Supplemental Fig. S3A). Having this ontogeny series in hand, we sought to test the therapeutic significance of the difference in cell of origin by subjecting the ontogeny series of primary cell cultures to cell cycle-specific inhibitors that we reasoned from the literature (Jahn et al. 1994; De Falco and De Luca 2006) may have differential sensitivity to cells of increasing myodifferentiation. The four cell cultures did indeed have differential sensitivities to the Cdk-2, Cdk-8, and Cdk-9 inhibitor SNS-032 and the Cdk-4/6 inhibitor PD0332991 (Fig. 3C,D). The primary tumor cell culture U23674 (Myf6Cre myoblast origin) was found to be the most sensitive to SNS-032 and PD0332991 compared with the other three primary tumor cell cultures (Pax7CreER 
Abraham et al.

A

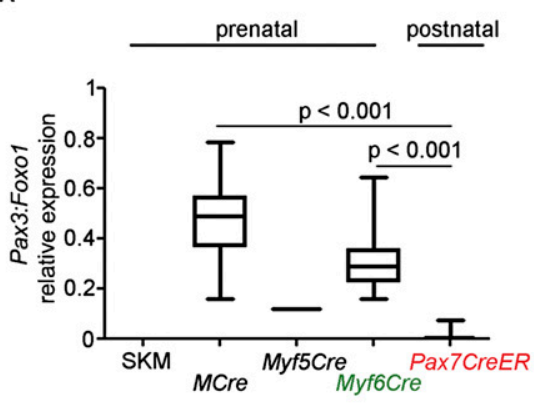

B

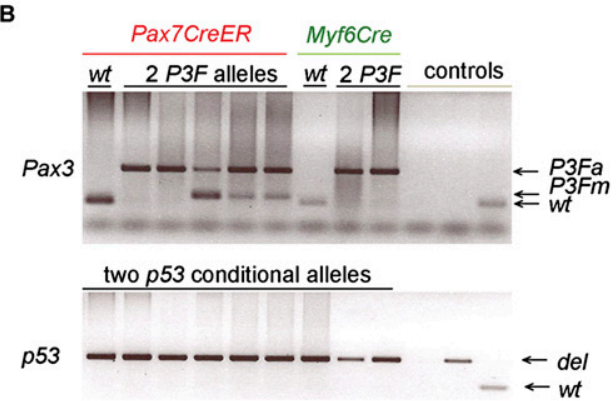

C
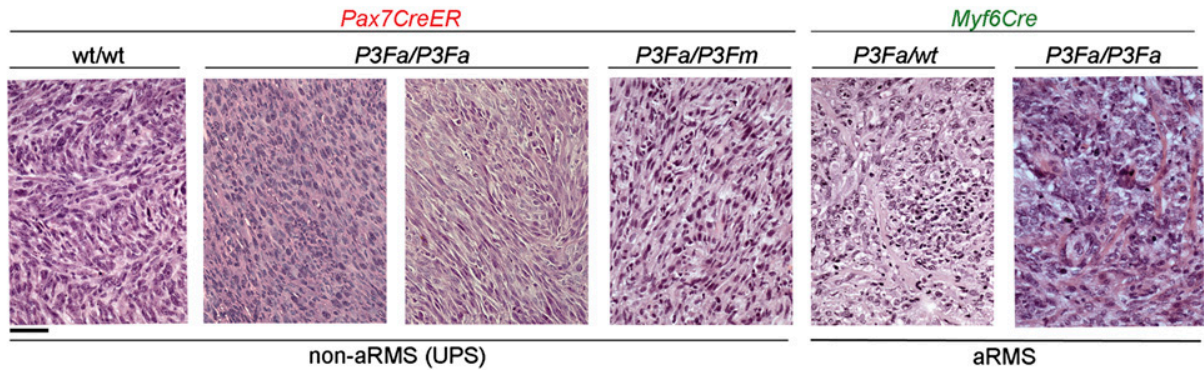

D

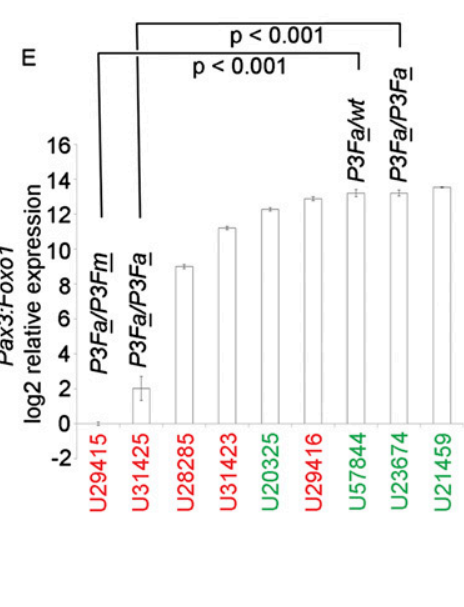

Figure 2. Recombination status of $P a x 3$ allele differs by cell of origin. $(A)$ Relative expression of Pax3:Foxo1 in mouse tumor tissue. (B) Genotyping for Pax3:Foxo1 and p53 recombination by cell of origin (Cre driver). Satellite cell-derived (Pax7CreER) primary tumor cell cultures showed reduced efficiency in the recombination of Pax3:Foxo1 knock-in allele locus compared with myoblast-derived primary tumor cell cultures (Myf6Cre). However, p53 locus conditional alleles were uniformly inactivated in all tumors irrespective of Cre driver. $(C)$ Representative H\&E staining of tumors with Cre driver and Pax3 locus genotypes as noted. All tumors had homozygous conditional $p 53$ deletion. Myf6Cre lineage tumors with heterozygous or homozygous activation of Pax3:Foxo1 showed small round cell histology consistent with aRMS. In contrast, Pax7CreER tumors with heterozygous or homozygous activation of Pax3:Foxo1 exhibited pleomorphic spindle cell appearances, similar to tumors with only p53 inactivation-neither of which appearance is consistent with aRMS. (D) Flow cytometry of eYFP for Pax7CreER-and Myf6Cre-derived primary tumor cell cultures. The mean florescence intensity was lower in Pax7CreER rhabdomyosarcoma cells, although select tumors of the Pax7CreER lineage (e.g., U31423 and U29416) more resembled Myf6Cre in eYFP expression (see the Results). (E) Quantitative RT-PCR (qRT-PCR) studies showing higher levels of Pax3:Foxo1 mRNA levels in Myf6Cre-derived primary tumor cell cultures (U23674 and U57844) compared with Pax7CreER-derived primary tumor cell cultures (U29415, U31425, and U31423). Statistically significant difference in Pax3:Foxo1 expression was observed between Myf6Cre- and Pax7CreER-derived primary tumor cell cultures with one activated Pax3:Foxo1(P3Fa) allele (U57844 and U29415) versus primary tumor cultures with two activated Pax3:Foxo1 alleles (U23674 and U31425).

satellite cell lineage), suggesting that the cell of origin of the sarcoma might play a role in deciding the treatment strategy for patients. Similar results were observed for the FGFR1-4 inhibitor, LY287445, and Wee1 inhibitor, MK1775, whereby Myf6Cre lineage-derived U23674 was found to be the most sensitive to these two drugs compared with intermediate ontogeny cultures U29416 and U31423 and Pax7CreER satellite cell lineage-derived U29415 (Supplemental Fig. S3C,D). Interestingly, when Pax3:Foxo1 was stably knocked down in U23674 cells 
A
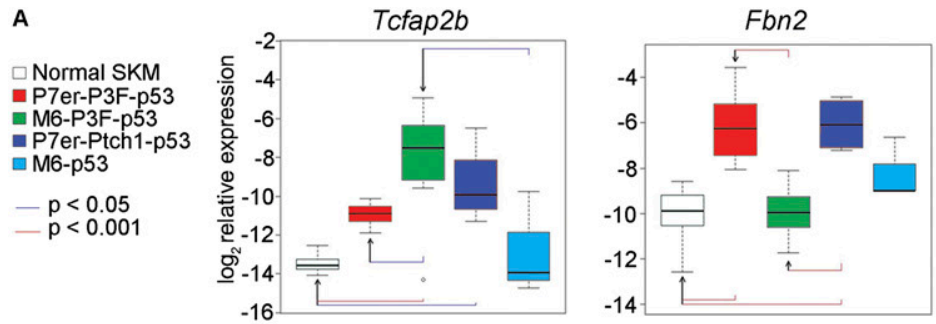

B

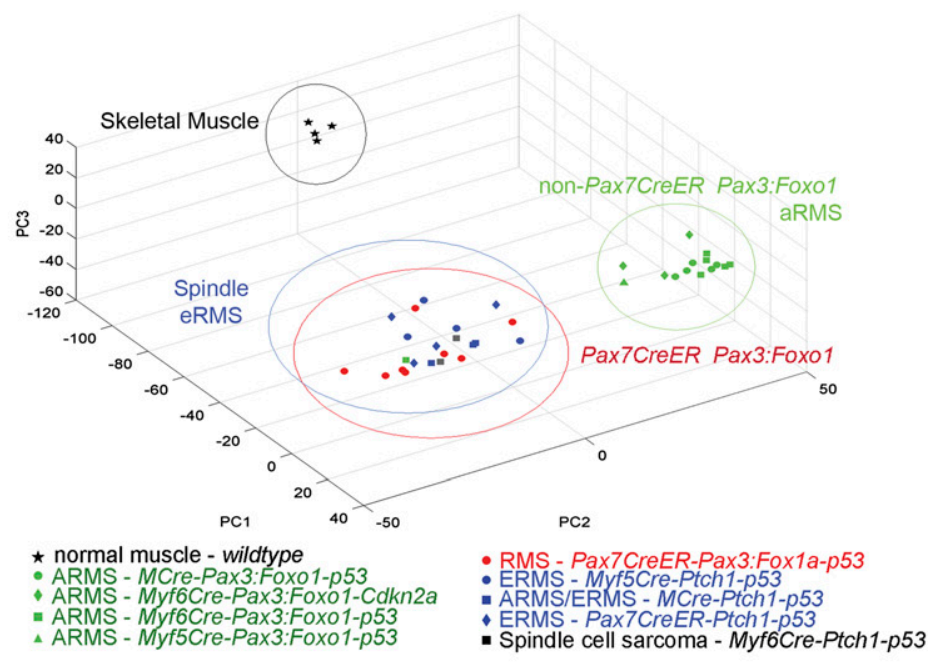

C

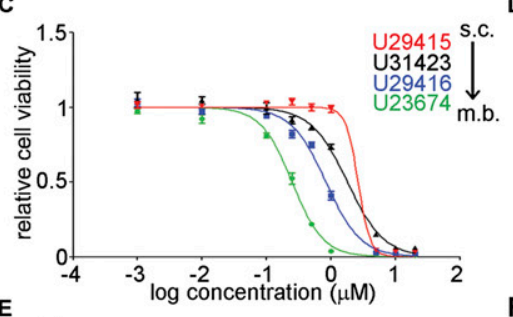

E

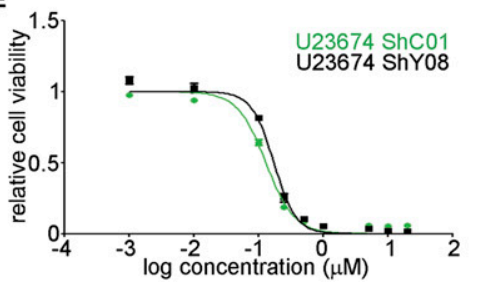

D

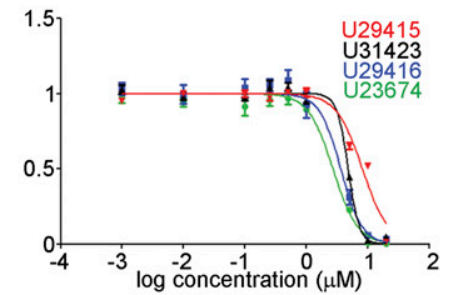

F

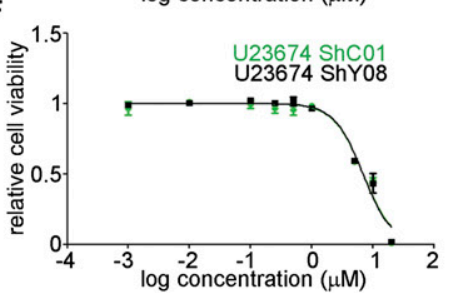

Figure 3. Pax3:Foxo1 and aRMS-specific marker expression in Pax3:Foxo1 tumors. $(A)$ For tumors bearing conditional Pax3:Foxo1 alleles, expression of aRMS-specific classifier Tcfap2b was significantly increased in Myf6Cre-derived tumors (M6-P3F-p53) and comparatively lower in Pax7CreER-derived tumors (P7er-P3F-p53); conversely, expression of eRMSspecific marker Fibrillin2 (Fbn2) was increased in Pax7CreER-derived, Pax3:Foxo1-bearing tumors and comparable with the level of Fbn2 from eRMS derived from satellite cells (P7er-Ptch1-p53) or myoblasts (M6-p53). (B) PCA showing the non-satellite cell-derived, Pax3:Foxo1-bearing tumors as an outgroup and Pax7CreER-derived, Pax3:Foxo1-bearing tumors grouping with spindle cell sarcoma/eRMS tumors bearing Ptch1 and p53 mutations. $(C, D)$ Cell viability assay showing differential drug response of primary tumor cell cultures along the continuum of satellite cell-derived (Pax7CreER) origin versus myoblast-derived (Myf6Cre). (C) For the Cdk-2, Cdk-7, and Cdk-9 inhibitor SNS-032, the IC50 values were $0.24 \mu \mathrm{M}$ for $\mathrm{U} 23674,0.83 \mu \mathrm{M}$ for $\mathrm{U} 29415,1.79 \mu \mathrm{M}$ for U31423, and 2.61 $\mu \mathrm{M}$ for U29415. (D) For the Cdk-4,-6 inhibitor PD0332991, the IC50 values were $2.69 \mu \mathrm{M}$ for $\mathrm{U} 23674,3.61 \mu \mathrm{M}$ for $\mathrm{U} 29415,4.77 \mu \mathrm{M}$ for $\mathrm{U} 31423$, and $8.15 \mu \mathrm{M}$ for $\mathrm{U} 29415$. $(E, F)$ Cell viability assay showing no difference in the response of U23674 ShY08 cells (Pax3:Foxo1 stable knockdown cells) to SNS-032 (E) and PD0332991 $(F)$ compared with the control knockdown cells, U23674 ShC01. For SNS-032, the IC50 values were $0.1305 \mu \mathrm{M}$ for ShC01 cells and $0.1730 \mu \mathrm{M}$ for ShY08 cells. In the case of PD0332991, the IC50 values were $6.90 \mu \mathrm{M}$ for ShC01 cells and $6.88 \mu \mathrm{M}$ for ShY08 cells. (s.c.) Satellite cell origin; (m.b.) myoblast origin.
(U23674 ShY08), there was no difference in their sensitivity to either SNS-032 (Fig. 3E) or PD0332991 (Fig. 3F) compared with the control cells (U23674 ShC01), further supporting that differential drug sensitivity was due to lineage of origin (i.e., epigenetic memory) and not Pax3:Foxo1 expression level.

The Pax7 and Pax3:Foxo1 loci are epigenetically regulated (differential effects of epigenetic modifiers on Pax3:Foxol and Pax7 expression depending on the tumor cell of origin)

We next reasoned that if tumor initiation and tumor phenotype is epigenetically determined, then the tumor phenotype can be epigenetically modified by pharmacological agents. The $P a x 7$ locus in activated satellite cells has a bivalent chromatin domain with the presence of both positive (H3K4me3) and negative (H3K27me3) histone marks (Mozzetta et al. 2011), and we hypothesized that the Pax3 locus may be similar. To gain insight into the epigenetic regulation of the Pax3:Foxo1 locus, we examined active (H3K4me3 and $\mathrm{H} 3 \mathrm{~K} 9 \mathrm{Ac})$ and repressive (H3K27me3 and H3K9me3) histone modification marks by chromatin immunoprecipitation (ChIP) in tumor cells representing both Pax7CreER (U29415 and U31425) and Myf6Cre (U23674 and U21459) lineages of origin. For this study, we analyzed two genomic regions upstream (-193: $-285)$ of and downstream $(+593:+462)$ from the transcrip- 
tional start site (TSS) of the Pax3 locus. At the level of histone marks, we observed a different chromatin state of the Pax3:Foxo1 locus in primary tumor cell cultures with different lineages of origin. As shown in Supplemental Figure S4, higher levels of both H3K4me3 and H3K9Ac active chromatin marks are observed in U23674 and U21459 cells compared with U29415 and U31425 tumor cells, correlating with high versus low Pax3:Foxo1 expression, respectively. Also high levels of repressive histone marks H3K27me3 and H3K9me3 were observed in U29415 and U31425 cells compared with U23674 and U21459 cells. Altogether, these data suggest that the observed differences in Pax3:Foxo1 mRNA levels could be explained by a different chromatin state in these loci depending on the tumor lineage of origin. From the RTPCR studies described above, U29415 was selected as a representative Pax7CreER lineage tumor culture, and U23674 was selected as a representative Myf6Cre lineage tumor culture.

To determine whether the Pax locus could be pharmacologically modified, we first treated murine primary tumor cell cultures U23674 and U29415 with varying doses of 5-Aza 2' deoxycytidine (DNA methyltransferase inhibitor) or SAHA and entinostat (histone deacetylase [HDAC] inhibitors) to determine a sublethal dose at which cells could be treated to study the effects of these drugs on expression of Pax3:Foxo1 and Pax7 (Supplemental Fig. S5). Cells were treated with drugs for 72 h, after which an MTS assay was performed to assess viability. Based on these studies, U23674 cells were treated with $3 \mu \mathrm{M}$ 5-Aza-2' deoxycytidine, $2 \mu \mathrm{M}$ entinostat, or $0.75 \mu \mathrm{M}$ SAHA for $24 \mathrm{~h}$, after which RNA was extracted, and the expression of Pax3:Foxo1 and Pax7 was examined by RTPCR. For U23674 tumor cells of the Myf6Cre myoblast lineage, a decrease in both Pax7 and Pax3:Foxo1 mRNA levels upon treatment with all of the drugs was observed, but the effect was more pronounced for HDAC inhibitors (Fig. 4A). Interestingly, U29415 tumor cells of Pax7CreER satellite cell lineage showed a paradoxical increase in mRNA levels of $P a x 7$ and Pax3:Foxo1 when treated with $20 \mu \mathrm{M}$ 5-Aza-2'deoxycytidine, $15 \mu \mathrm{M}$ entinostat, and $5 \mu \mathrm{M}$ SAHA (Fig. 4A). As expected, the rare tumor cultures downstream from U29415 behaved similarly to the Myf6Cre tumor cultures (Supplemental Fig. S6). We next determined epigenetic marks of Pax3:Foxo1 by performing ChIP and examining the active histone marks H3K4me3 and H3K9Ac along with the repressive marks H3K27me3 and H3K9me3 previously examined in Supplemental Figure S4. However, examination of these specific chromatin marks did not explain the change in Pax3:Foxo1 transcript levels, implying that other regions may be the targets of chromatin remodeling for entinostat.

Previous studies in $m d x$ mice treated with a TNF- $\alpha$ antibody reported increased levels of $\operatorname{Pax} 7$ mRNA in satellite cells derived from myofibers (Palacios et al. 2010). TNF- $\alpha$ has been shown to activate p38 $\alpha$ kinase, which leads to interaction between YY1 and PRC2 (polycomb-repressive complex 2) via threonine phosphorylation of EZH2. These proteins together form a repres- sive complex at the $\operatorname{Pax} 7$ promoter and thus regulate the expression of Pax7 (Palacios et al. 2010). To investigate whether TNF- $\alpha$ can regulate the expression of $\mathrm{Pax} 7$ in U23674 and U29415 tumor cells, cultures were treated with $10 \mathrm{ng} / \mathrm{mL}$ recombinant TNF- $\alpha$ for $24 \mathrm{~h}$. RT-PCR data showed a reduction in mRNA levels of Pax7 in both U23674 and U29415 cells. TNF- $\alpha$ also reduced Pax3:Foxo1 levels in both cell cultures (Fig. 4A). We next examined the levels of Pax3:Foxo1 at the protein level. By immunoblotting, concomitant reduction in Pax3:Foxol levels in $\mathrm{U} 23674$ cells treated with $2 \mu \mathrm{M}$ entinostat for $24 \mathrm{~h}$ was observed (Fig. 4B). This effect was seen across species for human aRMS cell lines $\mathrm{Rh} 30$ ( $24 \mathrm{~h}$ of treatment) and Rh41 (24 h of treatment) (Fig. 4C,D). When murine and human tumor cultures were treated with $2 \mu \mathrm{M}$ entinostat for $72 \mathrm{~h}$, a pronounced reduction in Pax3:Foxol protein levels was observed (Fig. 4E,F). These results showed in principle that Pax3:Foxo1 and Pax7 transcription and protein levels can be modified pharmacologically. To investigate whether the reduction in Pax3:Foxol protein levels upon entinostat treatment is mediated by changes in post-translational stability attributable to direct HDACPax3:Foxo1 interactions, we treated human and murine aRMS cultures (Rh41 for $24 \mathrm{~h}$ and U23674 for $72 \mathrm{~h}$ ) with 2 $\mu \mathrm{M}$ entinostat alone or in combination with MG132 (proteasome inhibitor). No increase in Pax3:Foxol protein level was observed when cells were treated with a combination of entinostat and MG132 compared with cells treated with entinostat alone (Fig. 4G,H). This result suggests that the reduction in Pax3:Foxol levels observed upon entinostat treatment is mediated at the transcriptional (or post-transcriptional) level and not at the post-translational level. Other studies from our laboratory have shown that expression of Pax3:Foxo1 is $G_{2}$ cell cycle phase-dependent (Kikuchi et al. 2014). To investigate whether the reduction in Pax3:Foxo1 expression caused by entinostat treatment is cell cycle-dependent, we performed a series of studies suggesting that this was not necessarily the case (Supplemental Fig. S7).

\section{Entinostat slows tumor growth in vivo}

We next tested the anti-tumor efficacy of entinostat in an orthotopic allograft model of rhabdomyosarcoma generated by injecting U23674 cells into the cardiotoxinpreinjured gastrocnemius muscle in the right leg of SCID-hairless (SHO-Prkdscid $\mathrm{Hr}^{\text {hr }}$ ) mice. Once the tumors reached $0.25 \mathrm{~cm}^{3}$, the mice were treated with a daily dose of $10 \mathrm{mg} / \mathrm{kg}$ entinostat by intraperitoneal injection, with $0.25 \mathrm{mg} / \mathrm{kg}$ actinomycin $\mathrm{D}$ by intraperitoneal injection on day 1 of treatment, a combination of entinostat and actinomycin D, or vehicle (DMSO) (Fig. 5A,B). Actinomycin D is one of the commonly used chemotherapeutic agents for treating rhabdomyosarcoma. The tumors in the entinostat cohort grew significantly slower than either vehicle- or actinomycin D-treated mice $(P=$ $0.03)$. Mice treated with a combination of entinostat and actinomycin D had a significantly slower rate of tumor growth, and their tumors took more time to reach $1 \mathrm{~cm}^{3}$ 
A
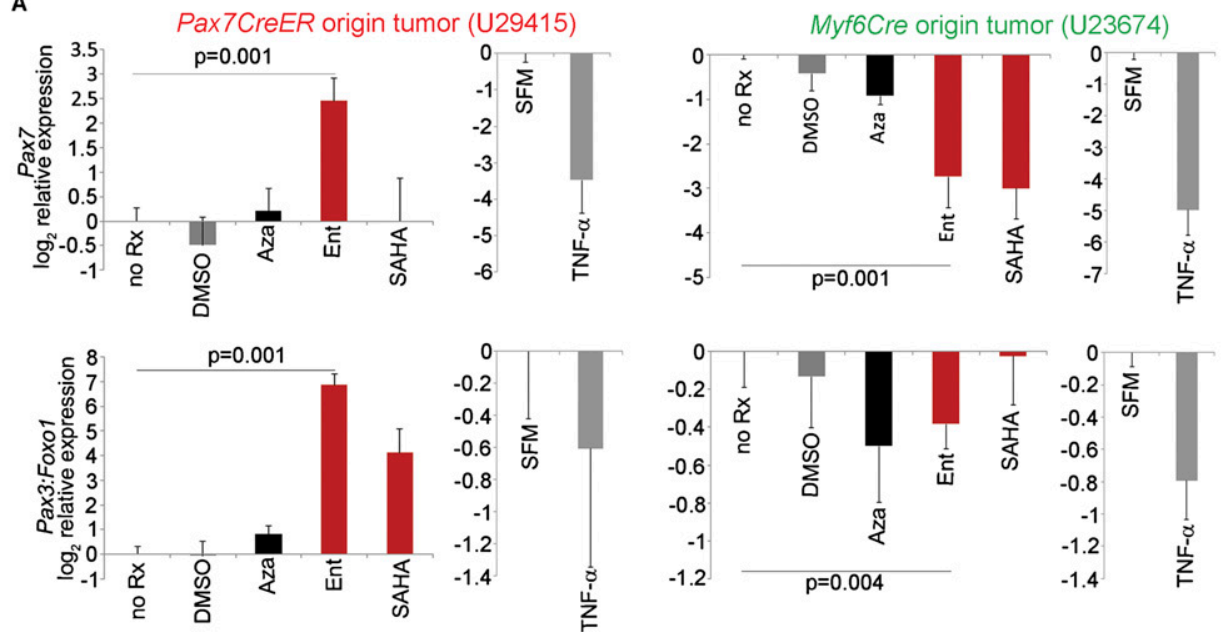

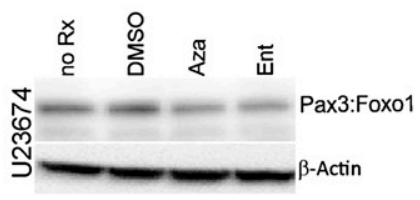

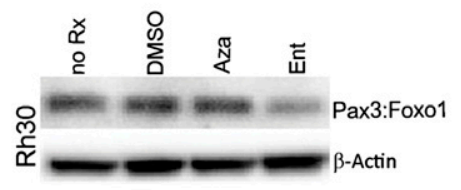

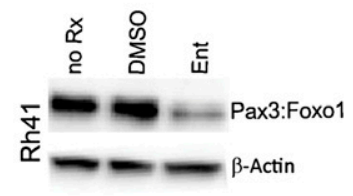

$\mathbf{E}$ $\mathbf{F}$

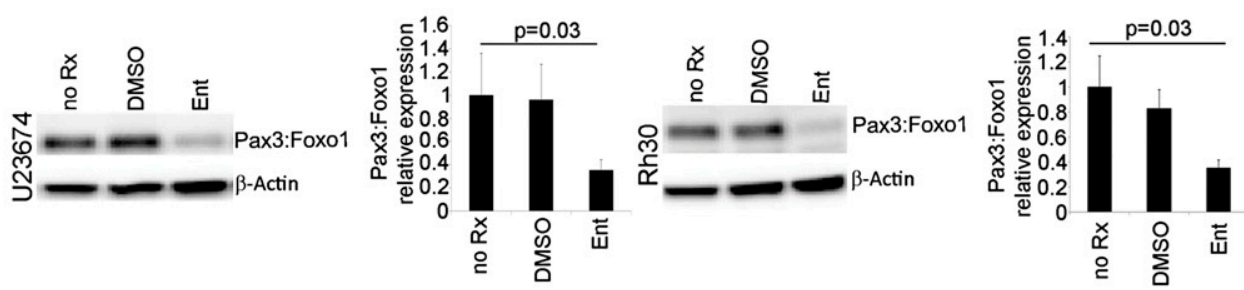

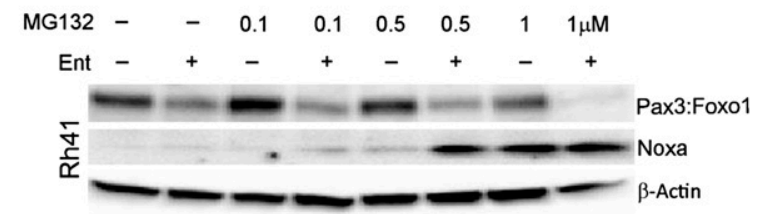

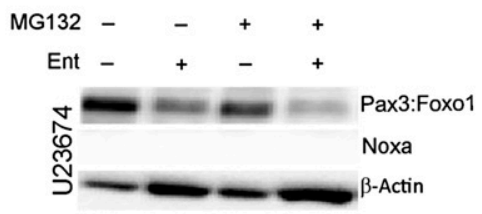

Figure 4. Pharmacologically modified epigenetic regulation of Pax3:Foxo1. (A) RT-PCR assays showing relative expression of Pax3:Foxo1 and Pax7 in representative satellite cell-derived U29415 and myoblast-derived U23674 murine primary tumor cell cultures upon treatment with DMSO, 5-Aza-2'deoxycytidine (Aza), SAHA, entinostat (Ent), serum-free medium (SFM), and the cytokine TNF- $\alpha$ (10 ng/mL in serum-free medium) for $24 \mathrm{~h}$. The U29415 primary tumor cell culture (satellite cell origin) showed an increase in Pax 7 and Pax3:Foxo1 expression upon treatment with 5-Aza-2'deoxycytidine, SAHA, and entinostat for $24 \mathrm{~h}$, whereas the U23674 primary tumor cell culture (myoblast origin) showed a decrease in the mRNA levels of Pax7 and Pax3:Foxo1 upon treatment. However, treatment with TNF- $\alpha$ showed a reduction in the expression of Pax7 and Pax3:Foxo1 in both U29415 and U23674 cells. (B) Immunoblot showing reduction in Pax3:Foxol levels upon treatment with $2 \mu \mathrm{M}$ entinostat (Ent) and also with $3 \mu \mathrm{M}$ 5-Aza-2'deoxycytidine (Aza) for $24 \mathrm{~h}$ in U23674 cells. (C) Reduction in Pax3:Foxol level upon treatment with $2 \mu \mathrm{M}$ entinostat (Ent) for $24 \mathrm{~h}$ in Rh30 cells. (D) Western blot showing reduction in Pax3:Foxol levels upon treatment with $2 \mu \mathrm{M}$ entinostat (Ent) for $24 \mathrm{~h}$ in Rh41 cells. (E) Immunoblot showing significant reduction in Pax3:Foxo1 levels upon treatment with $2 \mu \mathrm{M}$ entinostat (Ent) for $72 \mathrm{~h}$ in U23674 cells (left panel) and the graphical representation (right panel). (F) Immunoblot showing a significant reduction in Pax3:Foxo1 level upon treatment with $2 \mu M$ entinostat (Ent) for $72 \mathrm{~h}$ in Rh30 cells (left panel) and the graphical representation for this blot (right panel). (G) Western blots showing no increase in Pax3:Foxol levels in Rh41 cells when they were treated with a combination of the proteasome inhibitor MG132 (0.1 $\mu$ M, $0.5 \mu \mathrm{M}$, and $1 \mu \mathrm{M})$ and $2 \mu \mathrm{M}$ entinostat for $24 \mathrm{~h}$ compared with cells treated with entinostat alone. Expression of Noxa was observed when cells were treated with a combination of MG132 and entinostat in Rh41 cells. $(H)$ Western blot showing no increase in Pax3:Foxo1 levels in U23674 cells even when these cells were treated with a combination of $200 \mathrm{nM}$ MG132 and $2 \mu \mathrm{M}$ entinostat for $72 \mathrm{~h}$ compared with cells treated with $2 \mu \mathrm{M}$ entinostat alone. Noxa could not be detected even after treating the cells with a combination of MG132 and entinostat for $72 \mathrm{~h}$. 
in size compared with vehicle-treated or actinomycin $\mathrm{D}$ alone-treated mice $(P<0.001)$ (Fig. 5 A,B). However, after $5 \mathrm{~d}$ of treatment with entinostat, the mice showed symptoms of drug toxicity ( $>10 \%$ loss of body weight), and hence no treatment was administered on day 6 , and the subsequent daily dose of entinostat was reduced to $5 \mathrm{mg} / \mathrm{kg}$.

\section{Discussion}

In our studies, we sought to refine experimentally the range of possibilities for the cell of origin of Pax3:Foxo ${ }^{+}$ rhabdomyosarcoma. Parenthetically, however, the cell of origin for Pax7:Foxo1 ${ }^{+}$aRMS is in no way addressed in this study. Surprisingly, in our experiments, the fetal myoblast (Myf6 lineage) appears most susceptible to formation of metastatic aRMS. In contrast, muscle stem cells (satellite cells) appear least poised to give rise to rhabdomyosarcoma-at least from adolescence onward. With regard to tumor initiation, our data suggest that the Pax3 chromatin structure may be closed (and relatively inaccessible to events like Cre/LoxP DNA recombination) and show that transcription from the Pax3 locus is inactive in tumor cells of this satellite cell lineage. However, this low transcriptional activity could be reversed by pharmacological epigenetic modifiers. Even more importantly, Pax3:Foxo1 can be reduced at the mRNA and protein level in myoblast-derived tumors by the same pharmacological epigenetic modifiers. The implication that cell of origin can influence pharmacological response is interesting, if not provocative. We concede, however, that common drug sensitivities are likely also found between tumors of different origins and that while some cell biological properties that may be transmitted from the cell of origin, as many or more may be the result of convergent, facultative phenotypes for tumor cell growth-even if rhabdomyosarcomas arise from different cell lineages.

What is remarkable is that a prenatal (Myf6Cre) lineage of origin should, in our comparison, be most capable of forming aRMS (at least in the context of p53 loss of function). Rhabdomyosarcoma is a disease that has a steady incidence throughout childhood (aRMS contrasts dramatically with eRMS, the latter of which peaks with toddler and adolescent growth spurts) (Ognjanovic et al. 2009). Suggestion of $\mathrm{Myf6}^{+}$prenatal or postnatal myogenic cells, possibly a terminally differentiating subpopulation, as an aRMS cell of origin was first reported by our group (Keller et al. 2004a,b) and later complemented by in vitro studies of human postnatal myoblasts transformed by PAX3:FOXO1 and other cooperative initiating mutations (Naini et al. 2008). Congenital fusion-positive aRMS is rarely reported, and congenital aRMS is generally fusion-negative (Godambe and Rawal 2000; Grundy et al. 2001; Sueters et al. 2005), although the PAX3:FOXO1 translocation has been detected in rare neonatal instances (Rodriguez-Galindo et al. 2001). What might account for the delay? One explanation might be an accumulation of other genetic changes (mutations), but epigenetic changes during childhood and adolescent growth and development might also have a role to play. In fact, epigenetic abnormalities are very common in human cancers-and, in contrast to genetic mutations, these epigenetic changes are potentially reversible.

Can cells other than those of skeletal muscle lineages give rise to aRMS? We are increasingly intrigued by this possibility, although the question needs to be addressed with prudence. A mesenchymal stem cell origin for aRMS has been suggested (Charytonowicz et al. 2009; Hettmer and Wagers 2010). In support of this possibility,
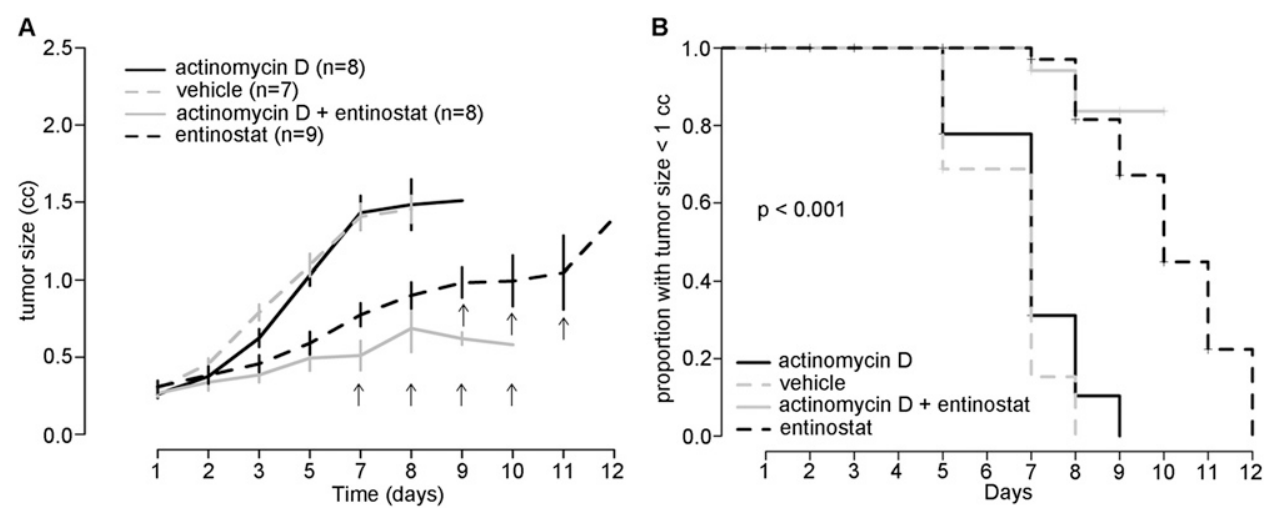

Figure 5. Anti-tumor activity of entinostat in an orthotopic allograft mouse mouse model of rhabdomyosarcoma. $(A)$ Tumor-bearing mice were treated with entinostat (dotted black line; $n=9 ; 10 \mathrm{mg} / \mathrm{kg}$ daily by intraperitoneal route), actinomycin $\mathrm{D}(\mathrm{black}$ line; $n=8$; $0.25 \mathrm{mg} / \mathrm{kg}$ on day 1 of treatment by intraperitoneal route), entinostat + actinomycin D (gray line; $n=8$ ), and DMSO (dotted gray line; $n=7)$. The graphs shows mean tumor size \pm SE by treatment and time. Tumor size increased monotonically with time after treatment with actinomycin D, DMSO, and entinostat and exhibited a downward trend after day 8 for the treatment group with actinomycin D + entinostat. The black arrows indicate the days on which entinostat- or entinostat + actinomycin D-treated mice were sacrificed for $10 \%-15 \%$ body weight loss. (B) Kaplan-Meier survival curves for tumor-bearing mice treated with entinostat daily, actinomycin D on day 1, entinostat + actinomycin $\mathrm{D}$, and vehicle (DMSO), with endpoint measurement being the number of days taken for the tumor to reach $1 \mathrm{~cm}^{3}$ in size. A null hypothesis that the treatment-specific survival curves are equal was tested using a log-rank test and concluded that the four curves are significantly different $(P<0.001)$. 
clinical cases of metastatic tumors without primary tumors have been reported for $t(1 ; 13)$ PAX7:FOXO1 aRMS (Lisboa et al. 2008) and t(2;13) PAX3:FOXO1 aRMS (Sandberg et al. 2001; Lisboa et al. 2008). An experimental study by Ren et al. (2008) tested the possibility that constitutively expressed (ectopic) Pax3:Foxo1 could create aRMS, but this study did not place Pax3:Foxo1 under the authentic control of the Pax3 promoter, which, in light of our studies of a very small $(0.4 \%)$ bone marrow compartment cell population that had ever expressed Pax3, makes the probability of aRMS arising from mesenchymal stem cells very low. However, a nonmarrow mesenchymal stem cell of origin remains possible.

An interesting alternative cell of origin for aRMS is the pericyte or mesangioblast. Vascular mimicry in rhabdomyosarcoma suggests a plasticity that may (or may not) have carried over from an angioblast origin (Sun et al. 2004). An interesting case of primary rhabdomyosarcoma of the pulmonary artery was also recently reported (Suman et al. 2013). Experimentally, Lagha et al. (2009) reported that in the mouse embryonic dermamyotome, the ratio of Pax3 to Foxc2 balances fate between myogenic and vascular cell fates but that Pax3:Foxol suppresses Foxc2. This latter result suggests that, in the susceptible cell, an angiogenic precursor might be converted to a myogenic state, a result that has been shown experimentally for embryonic aortic mesangioblasts (Messina et al. 2009). The link between vascular precursors and the Pax3 lineage does not come as a surprise given the known role of Pax3 in the migration of cardiac neural crest during embryogenesis (Conway et al. 1997). In a provocative study, Goupille et al. (2011) have shown that smooth muscle cells of arteries express Pax3 and are able to contribute to skeletal muscle fiber formation via in vitro coculture conditions and following cell-cell fusion. This plasticity lends itself to the possibility that mesangioblasts or even smooth muscle cells can transform into myogenic phenotype cancers (rhabdomyosarcomas) under select conditions.

Our results raise a potentially important clinical implication by suggesting that $P A X 3: F O X O 1$ levels vary by cell of origin, even adjusting for recombination frequency; thus, PAX3:FOXO1 RT-PCR alone may be less sensitive than cytogenetics or FISH to detect the PAX3:FOXO1 rearrangements. Could some MFH or UPS cases be in fact a spindle cell variant of aRMS, arising from satellite cells? Pathologists might consider addressing this possibility in cases of pediatric and adolescent undifferentiated sarcomas and consider FISH for PAX3:FOXO1 in atypical cases.

Translationally, our data suggest a mechanism by which fusion-positive aRMS might be epigenetically reprogrammed into fusion-negative RMS using the adult phase II HDAC inhibitor entinostat. Transcription factors were once considered undruggable, but our approach complements other experimental demonstrations for EWS:FLI1 in pediatric Ewing's sarcoma (Barber-Rotenberg et al. 2012) and MLL-associated pediatric leukemias (Chen et al. 2013). While additional preclinical studies remain to be done, it is exciting to consider that entinostat might reduce $P A X 3: F O X O 1$ levels sufficiently to convert fusion-positive RMS with a poor prognosis to a biological state akin to fusion-negative RMS, which portends a significantly better prognosis even when metastatic (Sorensen et al. 2002; Missiaglia et al. 2012).

\section{Materials and methods}

Microarray analysis for $S$ scores and PCA

Human microarray data sets were previously published (Wachtel et al. 2004; Lae et al. 2007; Davicioni et al. 2009). Patient demographics are presented in Supplemental Table 1, A and B. Metagene and S-score analyses were conducted as previously described (Rubin et al. 2011). For mouse tumors, gene expression analysis was performed using Illumina's Mouse Ref 8 Beadchip version 1 (Illumina, Inc.). These data sets have been deposited in Gene Expression Omnibus as accession entry GSE22520 and are also described in Supplemental Table 1C. Rank invariant normalization was performed on the $\log _{2}$ transformed expression value. Afterward, we applied PCA to all mouse tissue samples with the 19,070 probes (selected with the criterion of average $\log _{2}$ intensity $>5.5$ and standard deviation $>0.1$ ) and then plotted in three-dimensional space for visualization (Fig. 4B). Similar microarray data analysis and the PCA methods were previously described (Rubin et al. 2011). All bioinformatics tasks were performed with MATLAB/Bioinformatics Toolbox (MathWorks, Inc.) unless otherwise noted.

We downloaded the public domain data sets for fusionnegative RMS reported by Davicioni et al. (2006) from https:// array.nci.nih.gov/caarray/project/details.actionproject.experiment. publicIdentifier=trich-00099 as well as aRMS rhabdomyosarcoma data sets from previously published reports (Wachtel et al. 2004; Lae et al. 2007). These fusion-negative RMS and aRMS data sets were designated as the test samples, whereas normal skeletal muscle samples reported by Bakay et al. (2002) were used as the control group. We also downloaded signaturespecific data sets as described below. All of the studies were performed on an Affymetrix U133A array platform (Affymetrix). Sample IDs used are given in Supplemental Tables 1 and 2. Control samples of each examination are the eRMS samples derived from Rubin et al. (2011). We selected 10 high positive, 10 low positive, and 10 negative control samples for each examination, whereas gene-wise $t$-tests were performed between the 20 positive controls and normal skeletal muscle in order to apply to $\mathrm{S}$ score. S score is a subtype scoring method to quantify each sample's consistency. By using the $\mathrm{S}$ score, we can unambiguously identify sample status and the amplitude of pathways or biological processes that gene signatures have depicted. The detailed S-score method has been previously described (Rubin et al. 2011).

We used previously established gene lists (Rubin et al. 2011) to examine whether human fusion-negative RMS and aRMS tumors had evidence of p53 loss of function, Shh gain of function, $\mathrm{pRb}$ loss of function, or Ras activation. Gene signatures of p53 loss of function were derived from gene expression data sets in breast cancer (Miller et al. 2005). We also downloaded data sets for medulloblastoma samples known to exhibit a Shh gain-offunction signature (Thompson et al. 2006). We took homolog genes from $R b 1$ wild-type and homozygous $R b 1$-deleted fusionnegative mouse sarcomas to be $\mathrm{pRb}$ loss-of-function gene signature (Rubin et al. 2011). For Ras activation, we used gene lists for the activated Ras signature of zebrafish eRMS (Langenau et al. 2007). The details of obtaining gene signature of each case have been previously described (Rubin et al. 2011). 
Mice

All animal procedures were conducted in accordance with the Guidelines for the Care and Use of Laboratory Animals and were approved by the Institutional Animal Care and Use Committee (IACUC) at the University of Texas Health Science Center at San Antonio or Oregon Health and Science University. The Myf6Cre, Myf5Cre, Pax7Cre, MCre, Pax7CreER, Pax3:Foxo1, LUSEAP, LacZ reporter $\left(\right.$ Rosa $\left._{2}{ }^{\mathrm{tm} 1 \mathrm{Sor}}\right)$, Pax3-Cre $\left(\mathrm{Pax}^{\mathrm{CreKI}}\right)$, and conditional p53 mouse lines and corresponding genotyping protocols have been described previously (Soriano 1999; Marino et al. 2000; Jonkers et al. 2001; Keller et al. 2004a; Brown et al. 2005; Nishijo et al. 2009a,b). Myf5CreER have been reported (Biressi et al. 2013), and Myf6CreER mice will be reported in detail elsewhere but are also described in Supplemental Figure S2. For Pax7CreER, $M y f 5 C r e E R$, and Myf6CreER mice, tamoxifen was administered at P30 as previously reported (Keller et al. 2004a). Tumor-prone mice were visually inspected every $2 \mathrm{~d}$ for tumors because of the fulminant onset in these models. Genotyping protocols for Pax3:Foxo1 and p53 were performed as described (Keller et al. 2004a).

\section{Mouse primary tumor cell cultures}

Mouse primary tumor cell cultures were generated from mouse tumors as described previously (Abraham et al. 2011).

\section{Survival analysis and statistical methods}

Kaplan-Meier survival analysis of the mice was performed with the endpoint being the development of tumors (i.e., disease-free survival). Survival plots were created using the survival package in R. Relative expression of Pax3:Foxo1 values were compared using the Mann-Whitney test (Fig. 2A). The log-rank test (Fig. $3 \mathrm{~A})$ was used to determine the statistical significance $(P<0.05)$ using Systat 12 software (Systat Software, Inc.). The expression of Pax7 and Pax3:Foxo1 compared with corresponding reference groups was done using the Mann-Whitney test with the Hochberg correction for multiple testing (Fig. 4A). Statistical analyses were performed using R 3.0.1 (The R Foundation for Statistical Computing, http://www.r-project.org).

\section{RNA isolation and quantitative RT-PCR ( $q R T-P C R$ )}

RNA isolation and RT-PCR from mouse tumors were performed as previously described (Nishijo et al. 2009a; Rubin et al. 2011). Probe sets for mouse samples were Gapdh-Mm99999915_g1, Fbn2_Mm00515742_m1, and Tcfap2b_Mm00493468_m1. Statistical analysis was performed by Kruskal-Wallis test with Tukey's multiple testing correction. For drug treatment studies, the myoblast-derived primary tumor cell culture U23674 was treated with either $750 \mathrm{nM}$ SAHA, $3 \mu \mathrm{M}$ 5-Aza-2'deoxycytidine, or $2 \mu \mathrm{M}$ entinostat, and the satellite cell-derived primary tumor cell culture U29415 was treated with either $5 \mu \mathrm{M}$ SAHA, $20 \mu \mathrm{M}$ 5-Aza-2'deoxycytidine, or $15 \mu \mathrm{M}$ entinostat for $24 \mathrm{~h}$. After drug treatment, total RNA was isolated, and cDNA was synthesized as described above. The expression of Pax3:Foxo1 and Pax7 was determined by qRT-PCR using TaqMan primers and probes (mouse Pax7-Mm00834032_m1) on a StepOnePlus real-time PCR system from Applied Biosystems.

\section{Histology, immunohistochemistry, and immunochemistry}

For MyoD, Myogenin, and Desmin immunohistochemistry, staining was performed as previously described (Rubin et al. 2011). Immunofluorescence for GFP has also been described previously (Nishijo et al. 2009a). For immunocytochemistry, murine rhabdomyosarcoma primary tumor cell cultures (U23674) and human rhabdomyosarcoma cell lines (Rh41) were cultured in an eight-well CultureSlide (BD Falcon). The cells were washed with PBS, fixed with $4 \%$ paraformaldehyde, permeabilized with $0.25 \%$ Triton X-100, and incubated with the antibody overnight at $4{ }^{\circ} \mathrm{C}$. The primary antibodies used were mouse anti-Pax3 (R\&D Systems), rabbit anti-phospho CDC2-Y15, and rabbit antiphospho Histone H3 (Cell signaling Technology). After incubation, the cells were washed with PBS and incubated with fluorescein isothiocyanate-conjugated anti-mouse and anti-rabbit IgG (Invitrogen) for $1 \mathrm{~h}$. The cells were then examined by using a Zeiss LSM700 confocal microscope and Zen 2010 imaging software (Carl Zeiss).

\section{Flow cytometry and cell cycle analysis}

eYFP expression in mouse rhabdomyosarcoma cells and bone marrow aspirates was assessed by flow cytometry. Cells were stained with propidium iodide at $20 \mu \mathrm{g} / \mathrm{mL}$ for dead cell elimination, followed by analysis on a FACScan cytometer (Becton Dickinson). For cell cycle analysis, primary mouse tumor cell cultures were harvested, washed with PBS, and fixed in cold $70 \%$ ethanol for $20 \mathrm{~min}$. After fixation, the cells were washed with PBS and then incubated with RNase A and $20 \mu \mathrm{g} / \mathrm{mL}$ propidium iodide. The cells were then analyzed by using an Influx flow cytometer (Becton Dickinson) and FlowJo software (Tree star, Inc.).

\section{Western blotting}

Procedures have been described previously (Abraham et al. 2011). The following primary antibodies were used: goat antiFKHR (catalog no. sc-9808, Santa Cruz Biotechnology) to detect Pax3:Foxol and mouse anti-Noxa (catalog no. ab13654, Abcam).

\section{Drug sensitivity assays}

Murine rhabdomyosarcoma primary tumor cell cultures were plated in a 96-well plate at 2500 per well. After $24 \mathrm{~h}$, the cells were incubated with varying concentrations of entinostat (Selleckchem), SAHA (LC Laboratories), and 5-Aza-2'deoxycytidine (SigmaAldrich) for $3 \mathrm{~d}$. The cytotoxic effect of the drugs was then assessed by using CellTiter 96 AQueous One cell proliferation assay (MTS) and a BioTek Synergy 2 plate reader (BioTek).

\section{Stable knockdown of Pax3:Foxo1}

Murine primary tumor cell cultures with stable knockdown of Pax3:Foxo1 were generated as described earlier (Kikuchi et al. 2014).

\section{In vivo studies with entinostat}

Orthotopic allograft mouse models of rhabdomyosarcoma were generated as described earlier (Aslam et al. 2014). Once the tumors reached $0.25 \mathrm{~cm}^{3}$, the mice were treated with either entinostat (LC Laboratories) at a daily dose of $10 \mathrm{mg} / \mathrm{kg}$ by intraperitoneal injection, actinomycin D (Sigma-Aldrich) at 0.25 $\mathrm{mg} / \mathrm{kg}$ on day 1 by intraperitoneal injection, a combination of entinostat and actinomycin D, or vehicle (DMSO). Once the tumors reached $1.5 \mathrm{~cm}^{3}$ or the mice became sick $(10 \%-15 \%$ loss of body weight) due to drug toxicity, the mice were euthanized. After $5 \mathrm{~d}$ of treatment with entinostat, the mice showed signs of drug toxicity (loss of body weight), so the treatment was halted 
for a day and then continued with $5 \mathrm{mg} / \mathrm{kg}$ daily dose (half the initial dose). All of the drug studies in mice were performed after receiving approval from the IACUC at Oregon Health and Science University.

\section{Statistical analysis for in vivo studies with entinostat}

A repeated measures linear mixed effects model with a compound symmetric autocorrelation assumption to contrast treatments pairwise on day 7 with regard to tumor size was used. Day 7 was chosen for analysis because this was the last day with nearly complete data (actinomycin $\mathrm{D}, n=7$; actinomycin $\mathrm{D}+$ entinostat, $n=8$; DMSO, $n=7$; entinostat, $n=9$ ). The tumor size data were log transformed prior to analysis and corrected the $P$ values for multiple comparisons using the Tukey method.

\section{Acknowledgments}

We thank Rishi Rikhi and Noah Berlow for assistance with data analysis. This work was sponsored by training awards to K.N. and J.A. from the Scott Carter Foundation; support from Ministerio de Ciencia e Innovación (MICINN) (SAF2009-08128/SAF2012-37427) to M.S.; and National Cancer Institute awards 5R01CA133229 to C.K., 1R01CA154923 to D.M.L., and R01 AR062185 to T.A.R. Y.N.-A. is a FPU fellow (Ministerio de Educación, Cultura y Deporte), E.C. is a FPI fellow (MICINN), and A.T.M. was supported by National Institute of General Medical Sciences (NIGMS) MARC-U^STAR GM 07717 and NIGMS MBRS-RISE GM 60655. The Developmental Studies Hybridoma Bank was developed under the auspices of the Eunice Kennedy Shriver National Institute of Child Health (NICHD) and is maintained by the University of Iowa, Iowa City, IA.

\section{References}

Abraham J, Prajapati SI, Nishijo K, Schaffer BS, Taniguchi E, Kilcoyne A, McCleish AT, Nelon LD, Giles FG, Efstratiadis A, et al. 2011. Evasion mechanisms to Igflr inhibition in rhabdomyosarcoma. Mol Cancer Ther 10: 697-707.

Arndt CA, Crist WM. 1999. Common musculoskeletal tumors of childhood and adolescence. $N$ Engl J Med 341: 342-352.

Aslam MI, Abraham J, Mansoor A, Druker BJ, Tyner JW, Keller C. 2014. PDGFR $\beta$ reverses EphB4 signaling in alveolar rhabdomyosarcoma. Proc Natl Acad Sci 111: 6383-6388.

Bakay M, Chen YW, Borup R, Zhao P, Nagaraju K, Hoffman EP. 2002. Sources of variability and effect of experimental approach on expression profiling data interpretation. $B M C$ Bioinformatics 3: 4.

Barber-Rotenberg JS, Selvanathan SP, Kong Y, Erkizan HV, Snyder TM, Hong SP, Kobs CL, South NL, Summer S, Monroe PJ, et al. 2012. Single enantiomer of YK-4-279 demonstrates specificity in targeting the oncogene EWS-FLI1. Oncotarget 3: 172-182.

Biressi S, Bjornson CR, Carlig PM, Nishijo K, Keller C, Rando TA. 2013. Myf5 expression during fetal myogenesis defines the developmental progenitors of adult satellite cells. Dev Biol 379: 195-207.

Blum JM, Ano L, Li Z, Van Mater D, Bennett BD, Sachdeva M, Lagutina I, Zhang M, Mito JK, Dodd LG, et al. 2013. Distinct and overlapping sarcoma subtypes initiated from muscle stem and progenitor cells. Cell Reports 5: 933-940.

Brown CB, Engleka KA, Wenning J, Min Lu M, Epstein JA. 2005. Identification of a hypaxial somite enhancer element regulating Pax3 expression in migrating myoblasts and characterization of hypaxial muscle Cre transgenic mice. Genesis 41: 202-209.

Charytonowicz E, Cordon-Cardo C, Matushansky I, Ziman M. 2009. Alveolar rhabdomyosarcoma: is the cell of origin a mesenchymal stem cell? Cancer Lett 279: 126-136.

Chen L, Deshpande AJ, Banka D, Bernt KM, Dias S, Buske C, Olhava EJ, Daigle SR, Richon VM, Pollock RM et al. 2013. Abrogation of MLL-AF10 and CALM-AF10-mediated transformation through genetic inactivation or pharmacological inhibition of the H3K79 methyltransferase Dot11. Leukemia 27: 813-822.

Conway SJ, Henderson DJ, Copp AJ. 1997. Pax3 is required for cardiac neural crest migration in the mouse: evidence from the splotch (Sp2H) mutant. Development 124: 505-514.

Davicioni E, Finckenstein FG, Shahbazian V, Buckley JD, Triche TJ, Anderson MJ. 2006. Identification of a PAX-FKHR gene expression signature that defines molecular classes and determines the prognosis of alveolar rhabdomyosarcomas. Cancer Res 66: 6936-6946.

Davicioni E, Anderson MJ, Finckenstein FG, Lynch JC, Qualman SJ, Shimada H, Schofield DE, Buckley JD, Meyer WH, Sorensen $\mathrm{PH}$, et al. 2009. Molecular classification of rhabdomyosarcomagenotypic and phenotypic determinants of diagnosis: a report from the Children's Oncology Group. Am J Pathol 174: 550-564.

De Falco M, De Luca A. 2006. Involvement of cdks and cyclins in muscle differentiation. Eur J Histochem 50: 19-23.

Engleka KA, Gitler AD, Zhang M, Zhou DD, High FA, Epstein JA. 2005. Insertion of Cre into the Pax3 locus creates a new allele of Splotch and identifies unexpected Pax3 derivatives. Dev Biol 280: 396-406.

Godambe SV, Rawal J. 2000. Blueberry muffin rash as a presentation of alveolar cell rhabdomyosarcoma in a neonate. Acta Paediatr 89: 115-117.

Goupille O, Pallafacchina G, Relaix F, Conway SJ, Cumano A, Robert B, Montarras D, Buckingham M. 2011. Characterization of Pax3-expressing cells from adult blood vessels. J Cell Sci 124: 3980-3988.

Grundy R, Anderson J, Gaze M, Gerrard M, Glaser A, Gordon A, Malone M, Pritchard-Jones K, Michalski A. 2001. Congenital alveolar rhabdomyosarcoma: clinical and molecular distinction from alveolar rhabdomyosarcoma in older children. Cancer 91: 606-612.

Hatley ME, Tang W, Garcia MR, Finkelstein D, Millay DP, Liu N, Graff J, Galindo RL, Olson EN. 2012. A mouse model of rhabdomyosarcoma originating from the adipocyte lineage. Cancer Cell 22: 536-546.

Hawkins DS, Spunt SL, Skapek SX. 2013. Children's Oncology Group's 2013 blueprint for research: soft tissue sarcomas. Pediatr Blood Cancer 60: 1001-1008.

Hettmer S, Wagers AJ. 2010. Muscling in: uncovering the origins of rhabdomyosarcoma. Nat Med 16: 171-173.

Hettmer S, Liu J, Miller CM, Lindsay MC, Sparks CA, Guertin DA, Bronson RT, Langenau DM, Wagers AJ. 2011. Sarcomas induced in discrete subsets of prospectively isolated skeletal muscle cells. Proc Natl Acad Sci 108: 20002-20007.

Jahn L, Sadoshima J, Izumo S. 1994. Cyclins and cyclin-dependent kinases are differentially regulated during terminal differentiation of C2C12 muscle cells. Exp Cell Res 212: 297-307.

Jonkers J, Meuwissen R, van der Gulden H, Peterse H, van der Valk M, Berns A. 2001. Synergistic tumor suppressor activity of BRCA2 and p53 in a conditional mouse model for breast cancer. Nat Genet 29: 418-425.

Keller C, Arenkiel BR, Coffin CM, El-Bardeesy N, DePinho RA, Capecchi MR. 2004a. Alveolar rhabdomyosarcomas in conditional Pax3:Fkhr mice: cooperativity of Ink4a/ARF and Trp53 loss of function. Genes Dev 18: 2614-2626. 
Keller C, Hansen MS, Coffin CM, Capecchi MR. 2004b. Pax3:Fkhr interferes with embryonic Pax3 and Pax7 function: implications for alveolar rhabdomyosarcoma cell of origin. Genes Dev 18: 2608-2613.

Kikuchi K, Keller C. 2012. The not-so-skinny on muscle cancer. Cancer Cell 22: 421-422.

Kikuchi K, Hettmer S, Aslam MI, Michalek JE, Laub W, Wilky BA, Loeb DM, Rubin BP, Wagers AJ, Keller C. 2014. Cellcycle dependent expression of a translocation-mediated fusion oncogene mediates checkpoint adaptation in rhabdomyosarcoma. PLoS Genet 10: e1004107.

Lae M, Ahn EH, Mercado GE, Chuai S, Edgar M, Pawel BR, Olshen A, Barr FG, Ladanyi M. 2007. Global gene expression profiling of PAX-FKHR fusion-positive alveolar and PAXFKHR fusion-negative embryonal rhabdomyosarcomas. I Pathol 212: 143-151.

Lagha M, Brunelli S, Messina G, Cumano A, Kume T, Relaix F, Buckingham ME. 2009. Pax3:Foxc2 reciprocal repression in the somite modulates muscular versus vascular cell fate choice in multipotent progenitors. Dev Cell 17: 892-899.

Langenau DM, Keefe MD, Storer NY, Guyon JR, Kutok JL, Le X, Goessling W, Neuberg DS, Kunkel LM, Zon LI. 2007. Effects of RAS on the genesis of embryonal rhabdomyosarcoma. Genes Dev 21: 1382-1395.

Lisboa S, Cerveira N, Vieira J, Torres L, Ferreira AM, Afonso M, Norton L, Henrique R, Teixeira MR. 2008. Genetic diagnosis of alveolar rhabdomyosarcoma in the bone marrow of a patient without evidence of primary tumor. Pediatr Blood Cancer 51: 554-557.

Malempati S, Hawkins DS. 2012. Rhabdomyosarcoma: review of the Children's Oncology Group (COG) Soft-Tissue Sarcoma Committee experience and rationale for current COG studies. Pediatr Blood Cancer 59: 5-10.

Marino S, Vooijs M, van Der Gulden H, Jonkers J, Berns A. 2000. Induction of medulloblastomas in p53-null mutant mice by somatic inactivation of $\mathrm{Rb}$ in the external granular layer cells of the cerebellum. Genes Dev 14: 994-1004.

Messina G, Sirabella D, Monteverde S, Galvez BG, Tonlorenzi R, Schnapp E, De Angelis L, Brunelli S, Relaix F, Buckingham $\mathrm{M}$, et al. 2009. Skeletal muscle differentiation of embryonic mesoangioblasts requires pax3 activity. Stem Cells 27: 157164

Miller LD, Smeds J, George J, Vega VB, Vergara L, Ploner A, Pawitan Y, Hall P, Klaar S, Liu ET, et al. 2005. An expression signature for p53 status in human breast cancer predicts mutation status, transcriptional effects, and patient survival. Proc Nat1 Acad Sci 102: 13550-13555.

Missiaglia E, Williamson D, Chisholm J, Wirapati P, Pierron G, Petel F, Concordet JP, Thway K, Oberlin O, Pritchard-Jones $\mathrm{K}$, et al. 2012. PAX3/FOXO1 fusion gene status is the key prognostic molecular marker in rhabdomyosarcoma and significantly improves current risk stratification. I Clin Oncol 30: 1670-1677.

Mozzetta C, Consalvi S, Saccone V, Forcales SV, Puri PL, Palacios D. 2011. Selective control of Pax7 expression by TNF-activated p38 $\alpha$ /polycomb repressive complex 2 (PRC2) signaling during muscle satellite cell differentiation. Cell Cycle 10: 191-198.

Naini S, Etheridge KT, Adam SJ, Qualman SJ, Bentley RC, Counter CM, Linardic CM. 2008. Defining the cooperative genetic changes that temporally drive alveolar rhabdomyosarcoma. Cancer Res 68: 9583-9588.

Nishijo K, Chen QR, Zhang L, McCleish AT, Rodriguez A, Cho MJ, Prajapati SI, Gelfond JA, Chisholm GB, Michalek JE, et al. 2009a. Credentialing a preclinical mouse model of alveolar rhabdomyosarcoma. Cancer Res 69: 2902-2911.
Nishijo K, Hosoyama T, Bjornson CR, Schaffer BS, Prajapati SI, Bahadur AN, Hansen MS, Blandford MC, McCleish AT, Rubin BP, et al. 2009b. Biomarker system for studying muscle, stem cells, and cancer in vivo. FASEB I 23: 26812690.

Ognjanovic S, Linabery AM, Charbonneau B, Ross JA. 2009. Trends in childhood rhabdomyosarcoma incidence and survival in the United States, 1975-2005. Cancer 115: 42184226.

Ognjanovic S, Martel G, Manivel C, Olivier M, Langer E, Hainaut P. 2012. Low prevalence of TP53 mutations and MDM2 amplifications in pediatric rhabdomyosarcoma. Sarcoma 2012: 492086

Palacios D, Mozzetta C, Consalvi S, Caretti G, Saccone V, Proserpio V, Marquez VE, Valente S, Mai A, Forcales SV, et al. 2010. TNF/p38 $\alpha /$ polycomb signaling to Pax7 locus in satellite cells links inflammation to the epigenetic control of muscle regeneration. Cell Stem Cell 7: 455-469.

Ren YX, Finckenstein FG, Abdueva DA, Shahbazian V, Chung B, Weinberg KI, Triche TJ, Shimada H, Anderson MJ. 2008. Mouse mesenchymal stem cells expressing PAX-FKHR form alveolar rhabdomyosarcomas by cooperating with secondary mutations. Cancer Res 68: 6587-6597.

Rodriguez-Galindo C, Hill DA, Onyekwere O, Pin N, Rao BN, Hoffer FA, Kun LE, Pappo AS, Santana VM. 2001. Neonatal alveolar rhabdomyosarcoma with skin and brain metastases. Cancer 92: 1613-1620.

Rubin BP, Nishijo K, Chen HI, Yi X, Schuetze DP, Pal R, Prajapati SI, Abraham J, Arenkiel BR, Chen QR, et al. 2011. Evidence for an unanticipated relationship between undifferentiated pleomorphic sarcoma and embryonal rhabdomyosarcoma. Cancer Cell 19: 177-191.

Sambasivan R, Comai G, Le Roux I, Gomes D, Konge J, Dumas G, Cimper C, Tajbakhsh S. 2013. Embryonic founders of adult muscle stem cells are primed by the determination gene Mrf4. Dev Biol 381: 241-255.

Sandberg AA, Stone JF, Czarnecki L, Cohen JD. 2001. Hematologic masquerade of rhabdomyosarcoma. Am J Hematol 68: $51-57$.

Sorensen PH, Lynch JC, Qualman SJ, Tirabosco R, Lim JF, Maurer HM, Bridge JA, Crist WM, Triche TJ, Barr FG. 2002. PAX3-FKHR and PAX7-FKHR gene fusions are prognostic indicators in alveolar rhabdomyosarcoma: a report from the Children's Oncology Group. J Clin Oncol 20: 26722679.

Soriano P. 1999. Generalized lacZ expression with the ROSA26 Cre reporter strain. Nat Genet 21: 70-71.

Sueters M, Peek AM, Ball LM, Hogendoorn PC, Scherjon SA, de Keizer RJ, Schalij-Delfos NE. 2005. Prenatal detection of orbital rhabdomyosarcoma. Arch Ophthalmol 123: 276-279.

Suman KS, Sharma P, Singh H, Bal C, Kumar R. 2013. Primary rhabdomyosarcoma of pulmonary artery: 18F-FDG PET/CT for detecting recurrence in a rare tumor. Clin Nucl Med 38: e155-e156.

Sun B, Zhang S, Zhao X, Zhang W, Hao X. 2004. Vasculogenic mimicry is associated with poor survival in patients with mesothelial sarcomas and alveolar rhabdomyosarcomas. Int $J$ Oncol 25: 1609-1614.

Takahashi Y, Oda Y, Kawaguchi K, Tamiya S, Yamamoto H, Suita S, Tsuneyoshi M. 2004. Altered expression and molecular abnormalities of cell-cycle-regulatory proteins in rhabdomyosarcoma. Mod pathol 17: 660-669.

Thompson MC, Fuller C, Hogg TL, Dalton J, Finkelstein D, Lau CC, Chintagumpala M, Adesina A, Ashley DM, Kellie SJ, et al. 2006. Genomics identifies medulloblastoma subgroups 
that are enriched for specific genetic alterations. J Clin Oncol 24: 1924-1931.

Tiffin N, Williams RD, Shipley J, Pritchard-Jones K. 2003. PAX7 expression in embryonal rhabdomyosarcoma suggests an origin in muscle satellite cells. Br J Cancer 89: 327-332.

Vintersten K, Monetti C, Gertsenstein M, Zhang P, Laszlo L, Biechele S, Nagy A. 2004. Mouse in red: red fluorescent protein expression in mouse ES cells, embryos, and adult animals. Genesis 40: 241-246.

Wachtel M, Dettling M, Koscielniak E, Stegmaier S, Treuner J, Simon-Klingenstein K, Buhlmann P, Niggli FK, Schafer BW. 2004. Gene expression signatures identify rhabdomyosarcoma subtypes and detect a novel $\mathrm{t}(2 ; 2)(\mathrm{q} 35 ; \mathrm{p} 23)$ translocation fusing PAX3 to NCOA1. Cancer Res 64: 5539-5545.

Wachtel M, Runge T, Leuschner I, Stegmaier S, Koscielniak E, Treuner J, Odermatt B, Behnke S, Niggli FK, Schafer BW. 2006. Subtype and prognostic classification of rhabdomyosarcoma by immunohistochemistry. I Clin Oncol 24: 816822. 


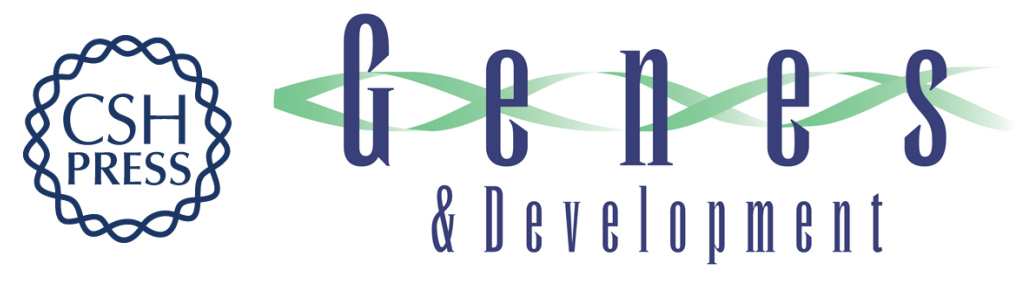

\section{Lineage of origin in rhabdomyosarcoma informs pharmacological response}

Jinu Abraham, Yaiza Nuñez-Álvarez, Simone Hettmer, et al.

Genes Dev. 2014, 28:

Access the most recent version at doi:10.1101/gad.238733.114

\section{Supplemental http://genesdev.cshlp.org/content/suppl/2014/07/16/28.14.1578.DC1 \\ Material}

References This article cites 60 articles, 19 of which can be accessed free at:

http://genesdev.cshlp.org/content/28/14/1578.full.html\#ref-list-1

Creative This article, published in Genes \& Development, is available under a Creative Commons

Commons License (Attribution 4.0 International), as described at

License http://creativecommons.org/licenses/by/4.0.

Email Alerting Receive free email alerts when new articles cite this article - sign up in the box at the top Service right corner of the article or click here.

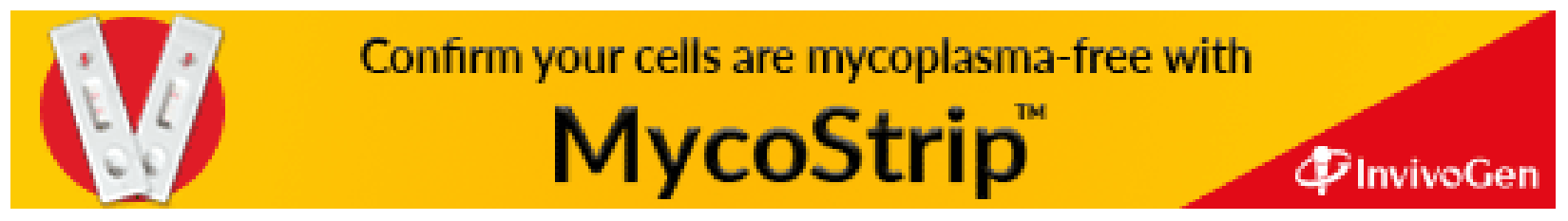

